\title{
ON THE STABILIZATION OF LINEAR SYSTEMS
}

\author{
C. E. LANGENHOP
}

1. In a recent paper $\mathrm{V}$. N. Romanenko [3] has given a necessary and sufficient condition that the system

$$
\frac{d x}{d t}=A x+b u, \quad \frac{d u}{d t}=p x+q u
$$

be "stabilizable." Here $A$ is an $n$ by $n$ matrix, $x$ and $b$ are $n$ by 1 column matrices (or vectors), $p$ is a 1 by $n$ row matrix and $q$ and $u$ are scalars. We shall assume that the elements of all these may be complex numbers. The vector $x$ can be interpreted physically as the output of a linear system characterized by the matrix $A$. The vector $b$ corresponds to some feedback or control mechanism with $u$ the controlling signal and $p$ and $q$ adjustable parameters in the controlling circuit. Romanenko calls the system $(A, b)$ stabilizable if for any nonempty set $S$ of $n+1$ or less complex numbers there exist $p$ and $q$ such that

$$
G=\left(\begin{array}{ll}
A & b \\
p & q
\end{array}\right)
$$

has $S$ as its set of characteristic values (spectrum). In particular then, if $(A, b)$ is stabilizable there exist $p$ and $q$ such that all characteristic values of $G$ have negative real parts and every solution of (1.1) is such that $x(t) \rightarrow 0$ and $u(t) \rightarrow 0$ as $t \rightarrow+\infty$.

In his paper Romanenko claims to be generalizing a known condition, which he attributes to $\mathrm{Yu}$. M. Berezanskir, namely, that if the characteristic values of $A$ are all distinct, then $(A, b)$ is stabilizable if and only if

$$
b, A b, \cdots, A^{n-1} b \text { are linearly independent. }
$$

Romanenko's condition appears to be considerably more complicated than (1.3) while it is our intention here to show that in fact (1.3) is necessary and sufficient for $(A, b)$ to be stabilizable irrespective of any condition on the characteristic values of $A$. Actually this is a corollary to our more general Theorem 1 given below.

As pointed out to the author by Dr. J. P. LaSalle the condition (1.3) and related ones have considerable significance in a seemingly

\footnotetext{
Presented to the Society, April 20, 1963 under the title Note on the stabilization of linear systems; received by the editors May 6, 1963.
} 
different aspect of control theory, namely, the controllability of linear dynamical systems [2]. Undoubtedly there is a deeper connection between stabilizability and controllability. At any rate a certain canonical form developed in the study of the latter concept provides a simplification of our original proof and a generalization of the Berezanskiľ-Romanenko result.

2. Rather than (1.2) we consider matrices of the form

$$
G=\left(\begin{array}{ll}
A & B \\
P & Q
\end{array}\right)
$$

where $A$ is $n$ by $n, Q$ is $m$ by $m$ and $B$ and $P$ are correspondingly sized submatrices. We assume that the elements of all these may be complex. By $S_{r}$ we denote a nonempty set of $r$ or less complex numbers $\mu_{k}$; that is, $S_{r}=\left\{\mu_{k} \mid k=1,2, \cdots, r\right\}$, where the $\mu_{k}$ need not all be distinct. The rank $h$ of the matrix $H=\left(B, A B, \cdots, A^{n-1} B\right)$ is the significant feature of our results, the first of which is

THEOREM 1. The condition $h \geqq r-m$ is necessary and sufficient that for each $S_{r}$ there exist $P$ and $Q$ such that the spectrum of $G$ contains $S_{r}$.

Proof of Necessity. Take any set $S_{r}$ such that the $\mu_{k}$ in $S_{r}$ are distinct and different from any characteristic value of $A$, and let $P$ and $Q$ be such that the spectrum of $G$ contains this $S_{r}$. Then there exist vectors

$$
\left(\begin{array}{l}
\xi_{k} \\
\eta_{k}
\end{array}\right), \quad k=1,2, \cdots, r,
$$

where $\xi_{k}$ and $\eta_{k}$ are $n$ by 1 and $m$ by 1 column matrices, respectively, such that

$$
\left(\begin{array}{ll}
A & B \\
P & Q
\end{array}\right)\left(\begin{array}{l}
\xi_{k} \\
\eta_{k}
\end{array}\right)=\mu_{k}\left(\begin{array}{l}
\xi_{k} \\
\eta_{k}
\end{array}\right)
$$

or

$$
\begin{aligned}
& A \xi_{k}+B \eta_{k}=\mu_{k} \xi_{k}, \\
& P \xi_{k}+Q \eta_{k}=\mu_{k} \eta_{k},
\end{aligned} \quad k=1,2, \cdots, r .
$$

From the first of (2.2) we may write

$$
\xi_{k}=\left(\mu_{k} I-A\right)^{-1} B \eta_{k}
$$

since $A-\mu_{k} I$ is nonsingular. Using the characteristic equation of $A$, one may write 


$$
\left(\mu_{k} I-A\right)^{-1}=\sum_{j=1}^{n} c_{j}\left(\mu_{k}\right) A^{j-1}
$$

for suitable scalar functions $c_{j}(\mu)$. Substituting (2.4) into (2.3), we have

$$
\xi_{k}=\sum_{j=1}^{n} A^{j-1} B c_{j}\left(\mu_{k}\right) \eta_{k}=H \zeta_{k}, \quad k=1,2, \cdots, r,
$$

where the transpose of the $n m$ by 1 column matrix $\zeta_{k}$ is defined by

$$
\zeta_{k}^{\prime}=\left(c_{1}\left(\mu_{k}\right) \eta_{k}^{\prime}, c_{2}\left(\mu_{k}\right) \eta_{k}^{\prime}, \cdots, c_{n}\left(\mu_{k}\right) \eta_{k}^{\prime}\right) .
$$

Now by choice of $S_{r}$ the vectors

$$
\left(\begin{array}{l}
\xi_{k} \\
\eta_{k}
\end{array}\right), \quad k=1,2, \cdots, r
$$

are linearly independent and it is readily seen that the rank of the matrix $\left(\xi_{1}, \xi_{2}, \cdots, \xi_{r}\right)$ must therefore be at least $r-m$. From (2.5) it is then clear that the rank of $H$ must likewise be at least $r-m$.

PROOF OF SUFFICIENCY. The proof is accomplished by means of a similarity transformation on $G$ to convert $A$ and $B$ to convenient canonical forms. We first dispose of the case $B=0$, however. In this event $h=0$ and the condition $h \geqq r-m$ becomes $m \geqq r$. It is clear then that we may choose $P$ arbitrarily and $Q$ such that the spectrum of $Q$ and hence also that of $G$ contains $S_{r}$. Henceforth, then, we assume $B \neq 0$.

Consider now a matrix $J$ of the form

$$
J=\left(\begin{array}{ll}
I & 0 \\
0 & R
\end{array}\right)
$$

where $I$ is an $n$ by $n$ identity and $R$ is a nonsingular $m$ by $m$ matrix. Then

$$
J G J^{-1}=\left(\begin{array}{cc}
A & B R^{-1} \\
R P & P Q R^{-1}
\end{array}\right)
$$

and it is evident that we may achieve any reordering of the columns of $B$ with no essential change in the statement of the proposition to be proved. Thus if the columns of $B$ are denoted by $b_{i}, i=1,2, \cdots, m$, we may assume without loss of generality that the set of $h$ columns of $H$

$$
b_{1}, A b_{1}, \cdots, A^{h_{1}-1} b_{1}, \cdots, b_{s}, A b_{\varepsilon}, \cdots, A^{h_{s}-1} b_{\imath}
$$


is linearly independent. Here $1 \leqq s \leqq m, h_{i} \geqq 1, i=1,2, \cdots, s$, and $\sum_{i=1}^{s} h_{i}=h$. We may further assume (see Chapter VII of [1]) that the sequence (2.8) is such that for $i \geqq 2$ the linear subspace $V_{i}$ spanned by $A^{j-1} b_{i}, j=1,2, \cdots, h_{i}$, is invariant modulo $V_{1}+V_{2}+\cdots+V_{i-1}$ under premultiplication by $A$. That is,

$$
A^{h_{i}} b_{i}=\sum_{j=1}^{h_{i}} \alpha_{i j} A^{j-1} b_{i}+\sum_{k=1}^{i-1} \sum_{j=1}^{h_{k}} \beta_{i j k} A^{j-1} b_{k}
$$

for some set of scalars $\alpha_{i j}, \beta_{i j k}$ and where the double summation in (2.9) does not appear if $i=1$.

We now introduce a similarity transformation on $G$ by means of a matrix $K$ of the form

$$
K=\left(\begin{array}{ll}
T & 0 \\
0 & I
\end{array}\right)
$$

where $I$ is an $m$ by $m$ identity and $T$ is a nonsingular $n$ by $n$ matrix. Then

$$
K G K^{-1}=\left(\begin{array}{cc}
T A T^{-1} & T B \\
P T^{-1} & Q
\end{array}\right)
$$

The matrix $T$ is defined by choosing certain combinations of the vectors in (2.8) as a new basis system for the space of $n$ by 1 column matrices. Thus we introduce

$$
e_{i k}=A^{h_{i}-k} b_{i}-\sum_{j=k+1}^{h_{i}} \alpha_{i j} A^{j-k-1} b_{i}, \quad \begin{aligned}
& k=1,2, \cdots, h_{i}, \\
& i=1,2, \cdots, s,
\end{aligned}
$$

and where the summation does not appear in case $k=h_{i}$. That is

$$
e_{i h_{i}}=b_{i}, \quad i=1,2, \cdots, s .
$$

Note from (2.12) and (2.13) we have for $k \geqq 2$

$$
A e_{i k}=e_{i, k-1}+\alpha_{i k} e_{i h_{i}}, \quad i=1,2, \cdots, s,
$$

and, using (2.9) in addition, we have, since the $A^{j-1} b_{i}$ 's may be expressed as linear combinations of the $e_{i j}$ 's,

$$
A e_{i 1}=\alpha_{i 1} e_{i h_{i}}+\sum_{k=1}^{i-1} \sum_{j=1}^{h_{k}} \gamma_{i j k} e_{k j}, \quad i=1,2, \cdots, s,
$$

for some set of scalars $\gamma_{i j k}$ and where, again, the double summation is absent in the case $i=1$.

From the linear independence of the set (2.8) and the remark just preceding (2.15) it is evident that the set 


$$
e_{11}, \cdots, e_{1 h_{1}}, e_{21}, \cdots, e_{2 h_{2}}, \cdots, e_{s 1}, \cdots, e_{s h_{s}}
$$

is likewise linearly independent. If $h=\sum_{i=1}^{s} h_{i}<n$ we may adjoin to the set (2.16) $n-h$ additional vectors to get a set which spans the space of $n$ by 1 matrices. We now view the matrix $A$ as defining the linear transformation $x \rightarrow A x$ and we let $T$ be defined so that for any $n$ by 1 matrix $x$ the matrix $T x$ is the column of components of $x$ relative to the set (2.16) (augmented if required) as basis. With respect to this basis the matrix of the linear transformation $x \rightarrow A x$ is the matrix $T A T^{-1}$ and, moreover, $T b_{i}=u_{\sigma_{i}}, \sigma_{i}=h_{1}+\cdots+h_{i}$ and $u_{\sigma}$ is an $n$ by 1 column all of whose components are zero except the $\sigma$ th which is 1. From (2.14) and (2.15) we may thus infer that $T A T^{-1}$ has the following block form:

$$
T A T^{-1}=\left(\begin{array}{ccccc}
C_{1} & E_{12} & \cdots & E_{1 s} & F_{1} \\
0 & C_{2} & \cdots & E_{2 s} & F_{2} \\
\cdot & \cdot & \cdot & \cdot \\
\cdot & \cdot & \cdot & \cdot \\
\cdot & \cdot & & \cdot & \cdot \\
0 & 0 & \cdots & C_{s} & F_{s} \\
0 & 0 & \cdots & 0 & L
\end{array}\right),
$$

where $C_{i}$ has the canonical form

Moreover,

$$
C_{i}=\left(\begin{array}{cccc}
0 & 1 & \cdots & 0 \\
\cdot & \cdot & & \cdot \\
\cdot & \cdot & & \cdot \\
\cdot & \cdot & & \cdot \\
0 & 0 & \cdots & 1 \\
\alpha_{i 1} & \alpha_{i 2} & \cdots & \alpha_{i h_{i}}
\end{array}\right) .
$$

$$
T B=\left(\begin{array}{lllll}
v_{1} & 0 & \cdots & 0 & w_{1} \\
0 & v_{2} & \cdots & 0 & w_{2} \\
\cdot & \cdot & & \cdot & \cdot \\
\cdot & \cdot & & \cdot & \cdot \\
\cdot & \cdot & & \cdot & \cdot \\
0 & 0 & \cdots & v_{s} & w_{s} \\
0 & 0 & \cdots & 0 & 0
\end{array}\right),
$$


where $v_{i}$ is an $h_{i}$ by 1 column all of whose components are zero except the last which is 1 . The submatrices $w_{i}$ have $m-s$ columns.

We now choose $P$ and $Q$ in convenient form. Let

$$
Q=\operatorname{diag}\left(q_{1}, q_{2}, \cdots, q_{m}\right)
$$

and let $P$ be such that

$$
P T^{-1}=\left(\begin{array}{ccccc}
p_{1} & 0 & \cdots & 0 & 0 \\
0 & p_{2} & \cdots & 0 & 0 \\
\cdot & \cdot & & . & \cdot \\
\cdot & \cdot & & \cdot & \cdot \\
\cdot & \cdot & & . & \cdot \\
0 & 0 & \cdots & p_{s} & 0 \\
0 & 0 & \cdots & 0 & M
\end{array}\right),
$$

where $p_{i}$ is a 1 by $h_{i}$ row and $M$ is $m-s$ by $n-h$. The determinant of $K G K^{-1}-\lambda I$ is now readily evaluated by means of Laplace expansions using minors from appropriate columns. Thus, if we expand by minors from the first $h_{1}$ columns along with the $(n+1)$ st column we find that only one of these is nonzero, namely, that using the first $h_{1}$ rows and the $(n+1)$ st row. The complementary minor will be a determinant array with exactly similar block structure to that of $K G K^{-1}-\lambda I$. It may thus be expanded similarly by using the corresponding columns, namely, those which appear in $K G K^{-1}-\lambda I$ as the second $h_{2}$ columns along with the $(n+2)$ nd. Continuing in this way we may write

$$
(2.21) \operatorname{det}\left(K G K^{-1}-\lambda I\right)=\left|\begin{array}{cc}
L-\lambda I & 0 \\
M & Q^{*}-\lambda I
\end{array}\right| \cdot \prod_{i=1}^{s}\left|\begin{array}{cc}
C_{i}-\lambda I & v_{i} \\
p_{i} & q_{i}-\lambda
\end{array}\right| \text {, }
$$

where $Q^{*}=\operatorname{diag}\left(q_{s+1}, \cdots, q_{m}\right)$ and, of course, each appearance of $I$ denotes an identity of appropriate size.

First we observe that $Q^{*}$ may be chosen so that the spectrum of $G$ contains any prescribed set $S_{m-s}$. We next examine the determinants in the product factor in (2.21). Each of these has the same structure and may readily be evaluated by a Laplace expansion using minors from the last two rows. The result is

$$
\begin{aligned}
\left|\begin{array}{cr}
C_{i}-\lambda I & v_{i} \\
p_{i} & q_{i}-\lambda
\end{array}\right|= & (-\lambda)^{h_{i}+1}+\left(q_{i}-\alpha_{i h_{i}}\right)(-\lambda)^{h_{i}} \\
& +\sum_{j=2}^{h_{i}}\left(\alpha_{i, j-1}+q_{i} \alpha_{i j}-p_{i j}\right)(-\lambda)^{j-1}+\left(q_{i} \alpha_{i 1}-p_{i 1}\right)
\end{aligned}
$$


where the $p_{i j}, j=1,2, \cdots, h_{i}$, are the components of the row $p_{i}$. In this form it is evident that we may determine $q_{i}$ and the $p_{i j}$, $j=1,2, \cdots, h_{i}$, so that the coefficients of this polynomial are any we desire. Thus $q_{i}$ and $p_{i}$ may be determined so that this factor of $\operatorname{det}\left(K G K^{-1}-\lambda I\right)$ has any given collection of $h_{i}+1$ roots. This is true for each $i$ so using this and the fact mentioned earlier regarding the choice of $Q^{*}$ it is clear that we may specify $P$ and $Q$ so that $G$ contains in its spectrum any given nonempty set of $m-s+\sum_{i=1}^{s}\left(h_{i}+1\right)$ $=h+m$ or less complex numbers. Thus if $h \geqq r-m$ we see that for any $S_{r}$ there exist $P$ and $Q$ such that $G$ contains $S_{r}$ in its spectrum.

COROLlaRy 1. The condition $h=n$ is necessary and sufficient that for each $S_{n+m}$ there exist $P$ and $Q$ such that $G$ has $S_{n+m}$ as its spectrum.

PRoof. In any case $h \leqq n$, so if $r=n+m$, then the condition $h \geqq r-m$ is equivalent to $h=n$.

Corollary 2. Condition (1.3) is necessary and sufficient that $(A, b)$ be stabilizable.

Proof. This is Corollary 1 in the case $m=1$.

REMARK. Even in case $h<n$ there may exist $P$ and $Q$ such that all characteristic roots of $G$ have negative real parts. It is clear from (2.21) that this is the case provided the characteristic roots of $L$ have negative real parts which, in turn, is related to how the linear subspace complementary to that spanned by the columns of $H$ is associated with those characteristic roots of $A$ which have negative real parts. In any case this question does not appear to be directly answerable merely in terms of the rank of $H$.

3. In this section as an application of the results in $\$ 2$ we point out the relevancy of condition (1.3) to the behavior of a more complicated system of differential equations than (1.1). Thus we consider the system

$$
\begin{aligned}
\frac{d x}{d t} & =A x+b u, \\
\frac{d^{r+1} u}{d t^{r+1}} & =p x+\sum_{k=0}^{r} q_{k} \frac{d^{k} u}{d t^{k}}
\end{aligned}
$$

where $A, b, x, u, p$ are as before and $q_{k}, k=0,1,2, \cdots, r$, are scalars.

THEOREM 2. For any integer $r \geqq 0$, if condition (1.3) holds, then there exist $p, q_{k}, k=0,1, \cdots, r$, such that, for every solution of (3.1), $x(t) \rightarrow 0$ and $u(t) \rightarrow 0$ as $t \rightarrow+\infty$. 
Proof. Introduce variables $u_{k}$ by the relations $u_{0}=u$,

$$
u_{k}=\frac{d u_{k-1}}{d t}, \quad k=1,2, \cdots, r .
$$

Then (3.1) may be written in the equivalent matrix form

$$
\frac{d}{d t}\left(\begin{array}{c}
x \\
u_{0} \\
\cdot \\
\cdot \\
\cdot \\
u_{r-2} \\
u_{r-1}
\end{array}\right)=\left(\begin{array}{cccccccc}
A & b & 0 & 0 & \cdot & \cdot & \cdot & 0 \\
0 & 0 & 1 & 0 & \cdot & \cdot & \cdot & 0 \\
\cdot & \cdot & \cdot & \cdot & \cdot & \cdot & \cdot & \cdot \\
\cdot & \cdot & \cdot & \cdot & \cdot & \cdot & \cdot & \cdot \\
\cdot & \cdot & \cdot & \cdot & \cdot & \cdot & \cdot & \cdot \\
0 & 0 & 0 & 0 & \cdot & \cdot & \cdot & 1 \\
0 & 0 & 0 & 0 & \cdot & \cdot & \cdot & 0
\end{array}\right)\left(\begin{array}{c}
x \\
u_{0} \\
\cdot \\
\cdot \\
u_{r} \\
u_{r-2} \\
u_{r-1}
\end{array}\right)+\left(\begin{array}{c}
0 \\
0 \\
\cdot \\
\cdot \\
0 \\
\cdot
\end{array}\right)\left(u_{r}\right.
$$

This is the form of (1.1) with $\left(x^{\prime}, u_{0}, \cdots, u_{r-1}\right)^{\prime}$ playing the role of $x^{\prime}$ there, $u_{r}$ the role of $u,\left(p, q_{0}, \cdots, q_{r-1}\right)$ the role of $p$ and

$$
A^{*}=\left(\begin{array}{cccccccc}
A & b & 0 & 0 & . & . & . & 0 \\
0 & 0 & 1 & 0 & . & . & . & 0 \\
\cdot & \cdot & . & & & & & . \\
\cdot & \cdot & . & & & & . \\
\cdot & \cdot & . & & & & & . \\
0 & 0 & 0 & 0 & . & . & . & 1 \\
0 & 0 & 0 & 0 & . & . & . & 0
\end{array}\right) \text { and } b^{*}=\left(\begin{array}{l}
0 \\
0 \\
\cdot \\
\cdot \\
\cdot \\
0 \\
1
\end{array}\right)
$$

playing the roles of $A$ and $b$, respectively. From Corollary 2 $p, q_{0}, \cdots, q_{r}$ exist such that $x(t) \rightarrow 0, u_{k}(t) \rightarrow 0, k=0,1, \cdots, r$, as $t \rightarrow+\infty$ if $b^{*}, A^{*} b^{*}, A^{* 2} b^{*}, \cdots, A^{* n+r-1} b^{*}$ are linearly independent. From the form of $A^{*}$ and $b^{*}$ as given in (3.4) it is easy to verify that this is true if condition (1.3) holds.

Analogous applications of Corollary 1 may be made.

\section{REFERENCES}

1. F. R. Gantmacher, The theory of matrices, Vol. 1, Chelsea, New York, 1960.

2. R. E. Kalman, Y. C. Ho and K. S. Narendra, Controllability of linear dynamical systems, Contributions to Differential Equations 1 (1963), 189-213.

3. V. N. Romanenko, On a stabilization theorem, Dopovidi Akad. Nauk Ukraln. RSR 1962, 863-867.

SOUTHERN ILLINOIS UNIVERSITY 